\title{
Short Period M Giant Stars in the Hipparcos Catalogue
}

\author{
Chris Koen, Dave Laney \\ South African Astronomical Observatory, PO Box 9, 7935 Observatory, \\ South Africa
}

\begin{abstract}
A number of $\mathrm{M}$ giant stars with periods of the order of a few days were discovered by the Hipparcos mission. Similar stars may also have been found by other large-scale surveys. Parallaxes and colour indices of a sample of these stars were used to estimate their radii, temperatures and luminosities. It is shown that it is very unlikely that they are ellipsoidal or spotted variables, and that they are most likely pulsating in high overtone modes.
\end{abstract}

\section{Introduction}

Among the advantages of general photometric surveys is the possibility of finding variables of hitherto unsuspected types. The Hipparcos survey team found a few dozen $\mathrm{M}$ giant variables with surprisingly short periods, some less than 10 days. The OGLE collaboration has also published data showing fairly short period $(P<20 \mathrm{~d}$ or so) variations in some of the reddest stars in their observation fields. At least some of these stars are presumably $M$ giants. What causes the photometric variations in these short period M giant (SPMG) stars?

Aside from the obvious binary stars, most $M$ giant variables in the Hipparcos catalogue with periods shorter than 100 days are classified either as semiregular variables, or as "small amplitude red variables" (usually interpreted as meaning pulsating stars). Both of these types of stars usually have periods rather longer, and amplitudes substantially larger, than those of the SPMGs. Some of SPMG stars in the Hipparcos catalogue, particularly those with the shortest periods, remained unclassified.

In the OGLE survey, most of the SPMGs are classified as "miscellaneous", meaning periodic, but neither eclipsing nor pulsating. According to Udalski et al. (1994), the miscellaneous variables "... are usually red subgiants and giants - most likely spotted, chromospherically active stars". Olech (1996) considered the further possibility that these stars are ellipsoidal variables. It is noteworthy that if the stars are indeed chromospherically active, then such stars (FK Comae stars) are much more common than has been thought up to now (for example, the GCVS lists only four). This would also have important consequences for ideas about the rotation velocities of late type evolved stars.

A sample of fourteen stars with $P<10$ days, selected from the Hipparcos catalogue, will be considered below. 


\section{Cause of the Periodic Variations}

In the case of an ellipsoidal variable, the requirement that the binary separation be larger than the radius $R$ of the red giant star demands that

$$
M_{2}>3.34 \times 10^{-3} R^{3} P^{-2}-M_{1},
$$

where $P$ is the period (in days), and $M_{1}$ and $M_{2}$ are the masses (in solar units) of the $\mathrm{M}$ giant and the hypothesised companion. In the case of a spotted star, the minimum period $P_{\mathrm{s}}$ is that corresponding to rotation at the break-up speed; it is given by

$$
P_{\mathrm{s}}=0.12 M_{1}^{-1 / 2} R^{3 / 2}
$$

The assumption that $M_{1}=1.3$ appears reasonable, whereas the radius can be calculated from the surface brightness equation

$$
\log R=2.970-\log \pi-0.2 V+0.356(V-I)
$$

(Dumm \& Schild 1998). The required parallaxes, magnitudes, and colour indices are all given in the Hipparcos catalogue.

Table 1 shows the values of $R, M_{2}, P_{\mathrm{s}}$ and $P$ for the stars in our sample. The radii are typical of those determined for similar stars by interferometry (e.g. Dyck, Van Belle, \& Thompson 1998). It is abundantly clear that the stars cannot be either ellipsoidal variables or spotted rotators.

Table 1. The mass $M_{2}$ is the minimum companion mass if the star is an ellipsoidal variable; $P_{\mathrm{s}}$ is the minimum period (in days) if the star is a spotted rotator; and $Q_{1}$ and $Q_{2}$ are pulsation constants from two different formulae.

\begin{tabular}{crrrccc}
\hline HIP number & $R$ & $M_{2}$ & $P_{\mathrm{s}}$ & $P$ & $Q_{1}$ & $Q_{2}$ \\
\hline 10013 & 158 & 354 & 203 & 6.12 & .0035 & .0067 \\
19391 & 279 & 1363 & 472 & 7.28 & .0018 & .0038 \\
30185 & 91 & 1948 & 88 & 1.13 & .0015 & .0035 \\
37708 & 84 & 63 & 78 & 5.58 & .0082 & .0149 \\
50222 & 133 & 907 & 156 & 2.95 & .0022 & .0030 \\
50389 & 75 & 36 & 66 & 6.15 & .0108 & .0161 \\
52789 & 97 & 68 & 98 & 6.69 & .0079 & .0134 \\
60984 & 71 & 68 & 61 & 4.21 & .0079 & .0126 \\
66407 & 108 & 179 & 115 & 4.86 & .0049 & .0102 \\
67226 & 95 & 77 & 94 & 6.08 & .0075 & .0139 \\
89510 & 88 & 128 & 84 & 4.21 & .0058 & .0124 \\
91494 & 110 & 157 & 117 & 5.28 & .0052 & .0084 \\
98815 & 106 & 1423 & 111 & 1.67 & .0017 & .0059 \\
113410 & 70 & 25 & 60 & 6.62 & .0128 & .0270 \\
\hline
\end{tabular}

The period - mean density relation for pulsating stars is

$$
Q_{1}=P \sqrt{\frac{M}{R^{3}}}
$$




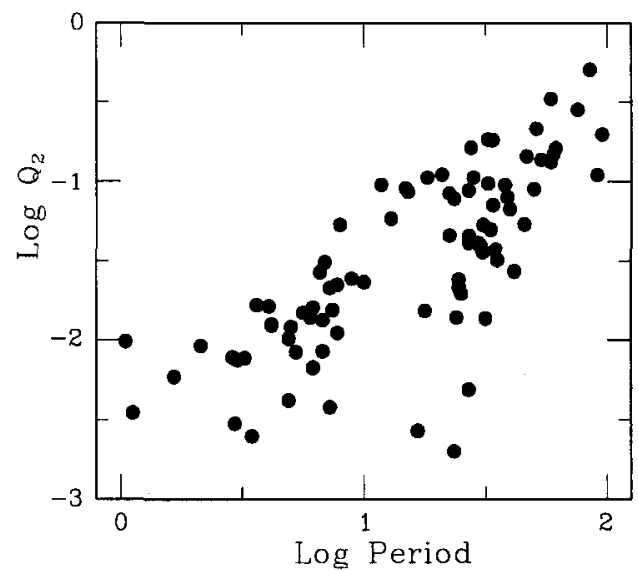

Figure 1. The pulsation constant $Q_{2}$ plotted against period for 85 Hipparcos $\mathrm{M}$ giants with $P<100$ days.

It is also possible to write this in a form which does not depend on the very uncertain value of the radius $R$ :

$$
\log Q_{2}=-12.71+\log P+0.5 \log M+0.3 M_{\text {bol }}+3 \log T_{\text {eff }} .
$$

The bolometric magnitude follows from the $V$ magnitude, parallax and a bolometric correction; and the effective temperature from the calibration

$$
\log T_{\text {eff }}=3.65-0.035(V-I)
$$

(Dumm \& Schild 1998). The last columns of Table 1 show the two pulsation constants for the stars; the values are all remarkably small.

\section{Concluding Remarks}

(1) Multiperiodicity has been found in a number of semiregular stars (e.g. Kiss et al. 1999). The presence of multiple periods in the observations would be a strong argument in favour of pulsation as the origin of the brightness variations. We tested the epoch photometry of the SPMG stars for multiple periods, and found some evidence for two or more periods in a number of the stars. However, the datasets are quite small and their time coverage very sparse, so that the results are uncertain.

(2) Fox \& Wood (1982) calculated pulsation constants for red giant stars. They found minimum values of $Q$ in the range $0.023-0.059$ for the fundamental, and for the first and second overtones. This suggests that the SPMG stars may be pulsating in very high overtones.

(3) The pulsation constant $Q_{2}$ is plotted against period in Fig. 1. Data for 85 Hipparcos M giant stars with $P<100$ days are shown. 
(4) If the overtone numbers of the SPMG stars are indeed large, we may expect to draw on roAp star research for ideas. For example, observations at different wavelengths may probe different phases of the pulsations - see Kurtz \& Medupe (1996) and Baldry et al. (1999) for discussions regarding the photometric and spectroscopic time series observations of pulsations in roAp stars. See also Laney \& Koen (2000).

(5) It appears that the SPMG data do not follow a clear-cut $P-L$ relation; in view of Fig. 1 this is not surprising.

(6) Line profile variations with similar periods have been found in $\mathrm{K}$ giant stars, and it appears likely that these are due to pulsation (e.g. Hatzes \& Cochran 1996). It is tempting to think of an instability region which includes both the $\mathrm{K}$ giants and the SPMG stars (which are mostly of early $M$ types). However, there seems to have been little evidence for photometric variability in K giant stars (see Percy 1993).

Acknowledgments. We are grateful for illuminating conversations with many of our colleagues. This research has made use of the Simbad database, operated at CDS, Strasbourg, France.

\section{References}

Baldry, I. K., Viskum, M., Bedding, T. R., Kjeldsen, H., \& Frandsen, S. 1999, MNRAS, 302, 381

Dumm, T. \& Schild, H. 1998, New Astronomy, 3, 137

Dyck, H. M., Van Belle, G. T., \& Thompson, R. R. 1998, AJ, 116, 981

Fox, M. W. \& Wood, P. R. 1982, ApJ, 259, 198

Hatzes, A. P. \& Cochran, W. D. 1996, in ASP Conf. Ser. Vol. 109, Cool Stars, Stellar Systems and the Sun, 9th Cambridge Workshop, ed. R. Pallavicini \& A. K. Dupree (San Francisco: ASP), 527

Kiss, L. L., Szatmáry, K., Cadmus Jr, R. R., \& Mattei, J. A. 1999, A\&A, 346, 542

Kurtz, D. W. \& Medupe, R. 1996, Bull. Astr. Soc. India, 24, 291

Laney, D. \& Koen, C. 2000, in these proceedings, p. 133

Olech, A. 1996, Acta Astron., 46, 389

Percy, J. R. 1993, PASP, 105, 1422

Udalski, A., Kubiak, M., Szymański, M., Kalużny, J., Mateo, M., \& Kremiński, W. 1994, Acta Astron., 44, 317

\section{Discussion}

Frank Pijpers: Is anything known about mass loss in these stars?

Chris Koen: The IRAS colors suggest that mass loss is insignificant for most of the stars with short periods. As they are of early $M$ spectral types, this is perhaps not surprising. 